\title{
Liquid Chromatography-Tandem Mass Spectrometric Assay for Determination of Stavudine in Human Plasma
}

\author{
Fengdan Jin \\ Key Laboratory of Applied Chemistry, Yanshan University, Qinhuangdao, Hebei 066004, China \\ Correspondence should be addressed to Fengdan Jin; fengdanjin@ysu.edu.cn
}

Received 10 March 2014; Revised 9 May 2014; Accepted 21 May 2014; Published 17 June 2014

Academic Editor: Bing Wu

Copyright (C) 2014 Fengdan Jin. This is an open access article distributed under the Creative Commons Attribution License, which permits unrestricted use, distribution, and reproduction in any medium, provided the original work is properly cited.

A LC-MS/MS method for determination of stavudine in human plasma was established and validated, and it was applied to the pharmaceutical formulations bioequivalence study. $0.5 \mathrm{~mL}$ plasma sample was extracted by liquid-liquid extraction. Stavudine was detected by a LC-MS/MS system. The pharmacokinetic parameters of stavudine in different formulations were calculated by noncompartment model statistics. The method was linear over the concentration ranges $5.00-1000 \mathrm{ng} / \mathrm{mL}$ in plasma. The intra- and interassay relative standard deviation (RSD) was $<10 \%$. The average accuracies for the assay at three concentrations $(5.00,80.0$, and $900 \mathrm{ng} / \mathrm{mL}$ ) were from $100.2 \%$ to $102.5 \%$. Pharmacokinetic parameters of stavudine reference formulation were obtained as follows: $T_{\max }$ was $0.6 \pm 0.2 \mathrm{~h}, C_{\max }$ was $480.7 \pm 150.9 \mathrm{~g} / \mathrm{L}, t_{1 / 2}$ was $1.7 \pm 0.4 \mathrm{~h}$, and $\mathrm{AUC}_{0-t}$ was $872.8 \pm 227.8 \mathrm{~g} \cdot \mathrm{h} / \mathrm{L}$, and pharmacokinetic parameters of stavudine test formulation were obtained as follows: $T_{\max }$ was $0.5 \pm 0.2 \mathrm{~h}, C_{\max }$ was $537.5 \pm 178.5 \mathrm{~g} / \mathrm{L}, t_{1 / 2}$ was $1.7 \pm 0.3 \mathrm{~h}$, and $\mathrm{AUC}_{0-t}$ was $(914.1 \pm 284.5) \mathrm{g} \cdot \mathrm{h} / \mathrm{L}$. Calculated with $\mathrm{AUC}_{0-t}$, the bioavailability of two formulations was $105.0 \%$.

\section{Introduction}

Stavudine is the first four approved for marketing the treatment of human immunodeficiency virus (HIV) infections following zidovudine, hydroxy glycosides, and go after zalcitabine by the U.S. Food and Drug Administration (FDA). It contains nucleoside reverse transcriptase inhibitors which can inhibit the replication of HIV-1 and HIV-2 in different kinds of cells. Stavudine triphosphate formed by cell kinase phosphorylation can prevent the HIV virus replication in human cells. Stavudine has been used in the adult HIVinfected people and AIDS patients who do not tolerate zidovudine and who can no longer be zidovudine-treated, and it also has been used in HIV-infected children from 3 months to 12 years old [1].

Determination of stavudine in plasma by high performance liquid chromatography method has been described $[2,3]$; analysis time was more than 12 minutes. The liquid chromatography-tandem mass spectrometry (LC-MS/MS) method [4] was established for the simultaneous monitoring of multiple anti-HIV drugs in vivo plasma concentrations after long-term combination therapy of AIDS. The LCMS/MS [5] method needs a larger workload because external standard was used. Two sets of standard curves and quality control (QC) samples were made to each analytical batch, and the system performance correction fluid was injected to confirm whether the response of ionization mass spectrometry is stable. This paper intends to establish a rapid, sensitive LCMS/MS method to determinate stavudine plasma concentrations for bioequivalence and pharmacokinetic study.

\section{Materials and Methods}

2.1. Reagents and Chemicals. Purity of stavudine and $\beta$ thymidine (internal standard) was more than $99.7 \%$ and 99.4\%. They were purchased from the Institute of Northeast General Pharmaceutical Factory (Shenyang, China). Zerit capsules were kindly supplied from Bristol-Myers Squibb Company (Canada). All chemicals and reagents were of analytical grade, and water was bidistilled water which was prepared from demineralized water.

2.2. Apparatus. Analyses were performed on an LC-MS/MS system composed of a Shimadzu LC-10AD pump (Kyoto, Japan), a Thermo Finnigan TSQ Quantum Ultra tandem 
mass spectrometer (San Jose, CA, USA), an LC-10ADVP pump, and a SIL-HTA autosampler.

2.3. Chromatographic Conditions. The analytical column was a Zorbax Extend C18 column $(150 \mathrm{~mm} \times 4.6 \mathrm{~mm}$, inner diameter, $5 \mu \mathrm{m}$; Agilent, Wilmington, DE, USA) and a SecurityGuard C18 guard column $(4 \mathrm{~mm} \times 3.0 \mathrm{~mm}$ inner diameter; Phenomenex, Torrance, CA, USA). The mobile phase was methanol-water $1 \%$ ammonia $(50: 50: 0.5, \mathrm{v} / \mathrm{v} / \mathrm{v})$. The liquid flow rate was set at $0.5 \mathrm{~mL} / \mathrm{min}$. The sample injection volume was $20 \mu \mathrm{L}$. The column temperature was maintained at $20^{\circ} \mathrm{C}$.

2.4. Mass Spectrometric Conditions. Stavudine and internal standard were monitored in the negative ion mode using a Turboion spray interface. The spray voltage was set at $3.8 \mathrm{kV}$ and the source temperature at $350^{\circ} \mathrm{C}$. Nitrogen was used as sheath gas $(20 \mathrm{Arb})$ and auxiliary gas $(2 \mathrm{Arb})$. The collision gas was argon at a pressure of 1.0 mTorr. Collision-induced dissociation voltage was $22 \mathrm{eV}$ for both the analyte and internal standard. The selected reaction monitoring (SRM) was selected to monitor precursor-product ion transitions. These corresponded to $m / z 223 \rightarrow m / z 42$ for stavudine and $m / z 241 \rightarrow m / z 42$ for internal standard. The dwell time was $0.3 \mathrm{~s}$ per transition.

2.5. Sample Preparation Procedure. A volume of $0.5 \mathrm{~mL}$ thawed human plasma was transferred to a $10 \mathrm{~mL}$ tube and $100 \mu \mathrm{L}$ of $\beta$-thymidine and $100 \mu \mathrm{L}$ methanol-water $(50: 50$, $\mathrm{v} / \mathrm{v})$ were spiked and briefly vortex-mixed. To each sample, $250 \mu \mathrm{L}$ phosphate buffer $(50 \mathrm{mmol} / \mathrm{L}, \mathrm{pH} 6)$ and $3 \mathrm{~mL}$ acetic ether were added. The tubes were capped and shaken for 10 minutes and then centrifuged at 3,500 rpm for 5 minutes. The upper organic phase was divided to a test tube and evaporated to dryness at $50^{\circ} \mathrm{C}$ by a gentle flow of air. The residue was dissolved in $100 \mu \mathrm{L}$ mobile phase, and a $20 \mu \mathrm{L}$ aliquot was injected into the LC-MS/MS system.

2.6. Bioequivalence and Pharmacokinetics Study. Cross double cycle test was adopted to invest bioequivalence study of two stavudine capsules. 20 healthy male volunteers were divided into two (I and II) groups stochastically, each group of 10 people. At the first cycle, group I and group II received the oral dosing of reference and text formulation (stavudine $20 \mathrm{mg}$ ), respectively. At the second cycle, groups I and II received the text and reference formulation, oppositely. Each test interval was for a week. Forearm venous blood $(3 \mathrm{~mL})$ was collected in tubes containing sodium heparin before taking the medicine $(0 \mathrm{~h})$ and at $0.25,0.50,0.75,1.0,1.5,2.0,3.0$, $4.0,6.0,8.0$, and $10.0 \mathrm{~h}$ after a single oral dose. The blood samples were centrifuged at $2,000 \mathrm{~g}$ for $10 \mathrm{~min}$ immediately. The plasma samples were separated and stored at $-20^{\circ} \mathrm{C}$ until analysis. Clinical trials were performed in Liaoning Province People's Hospital.

The plasma samples were prepared according to Section 2.5. One standard curve was established in each analytical batch, and double QC samples of three concentrations (high, medium and low) were analyzed, at the same time. The concentration data of the unknown sample and the QC sample were calculated by the standard curve.

\section{Results and Discussion}

3.1. Chromatographic and Mass Spectrometric Conditions. The selection of internal standard for LC-MS/MS method was different for HPLC-UV method. The ideal internal standard is isotope marker of analyte, but it is expensive and difficult to get, generally. Because of the instrument, the pressure of collision gas changes within a day. This leads the ratio of mass spectrometry signal intensity for analyte and internal standard to change. As a result, the intra-assay deviation of QC samples was large. Therefore, the requirement of internal standard is as follows: when the collision gas pressure changed, the signal intensity change of analyte and internal standard should trend accordingly. Zidovudine and $\beta$-thymidine were structural analogues of stavudine and were tried to be used as internal standard. In selected mass spectrometry conditions and chromatographic conditions, zidovudine response was high, but the retention time is $1 \mathrm{~min}$ longer than stavudine. $\beta$-thymidine also has a good response, and retention time was similar to stavudine, with good reproducibility. Considering the analysis time, $\beta$-thymidine was selected as the internal standard substance. The analyte and the internal standard formed predominately protonated molecules $[\mathrm{M}-\mathrm{H}]^{-}, m / z 223$ and $m / z$ 241. Through collisioninduced dissociation, and thire major fragment ions were both at $m / z 42$ (Figure 1).

3.2. Sample Preparation. Biological sample pretreatment method was improved as liquid-liquid extraction (LLE) which is most commonly used. LLE can remove fats, proteins, and endogenous ions effectively, and the operation is simple. LLE method lies in choosing the right extraction solvent and acidification and alkalization reagent according to the analyte polarity and acid-base property. Stavudine and $\beta$-thymidine are polar compounds, and both have acid group. The etherdichloromethane $(3: 2)$ and ethyl acetate were tried as the extraction solvent. The $\mathrm{pH} 4$ and $\mathrm{pH} 6$ phosphate buffer $(50 \mathrm{mmol} / \mathrm{L})$ were tried as acidifying agents. The ethyl acetate and $\mathrm{pH} 6$ phosphate buffer $(50 \mathrm{mmol} / \mathrm{L})$ were determined as extraction solvent and acidifying agents, because the extraction rate of analyte and the internal standard was higher (about $50 \%$ of both) and stable.

3.3. Selectivity. Blank plasma samples of six volunteers were prepared according to Section 2.5 (no internal standard), and the chromatogram was shown in Figure 2(a). A certain concentration of stavudine reference solution and internal standard solution was joined in blank plasma, with the same operation, and the chromatogram was shown in Figure 2(b). The retention time was $2.98 \mathrm{~min}$ and $2.83 \mathrm{~min}$, respectively. The chromatogram of plasma sample of a volunteer after oral dosing was shown in Figure 2(c). The results showed that the endogenous substances of blank plasma do not interfere with the determination of the analytes and internal standard. 


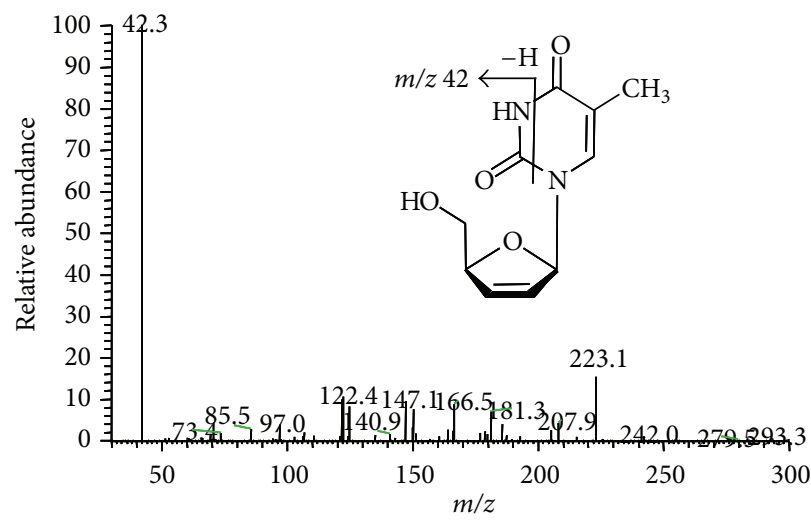

(a)

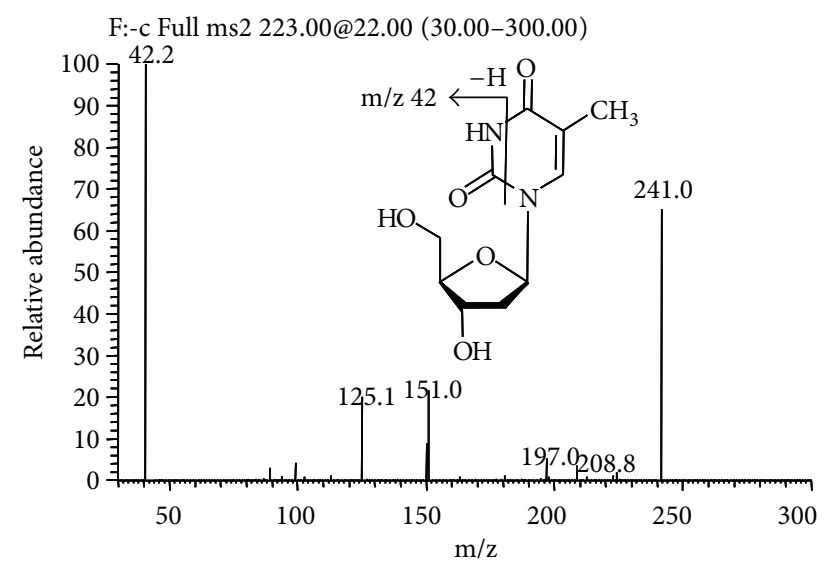

(b)

FIGURE 1: Full-scan product ion spectra of $[\mathrm{M}-\mathrm{H}]^{-}$of (a) stavudine and (b) $\beta$-thymidine (internal standard).

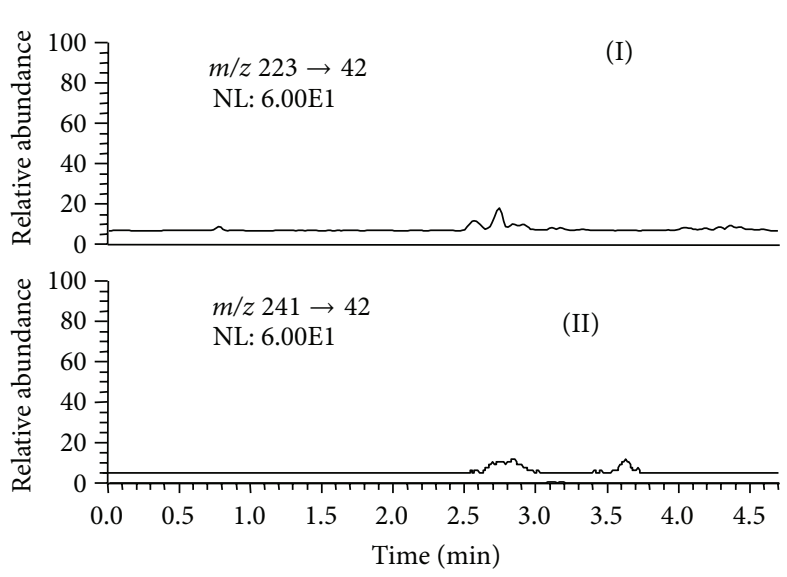

(a)

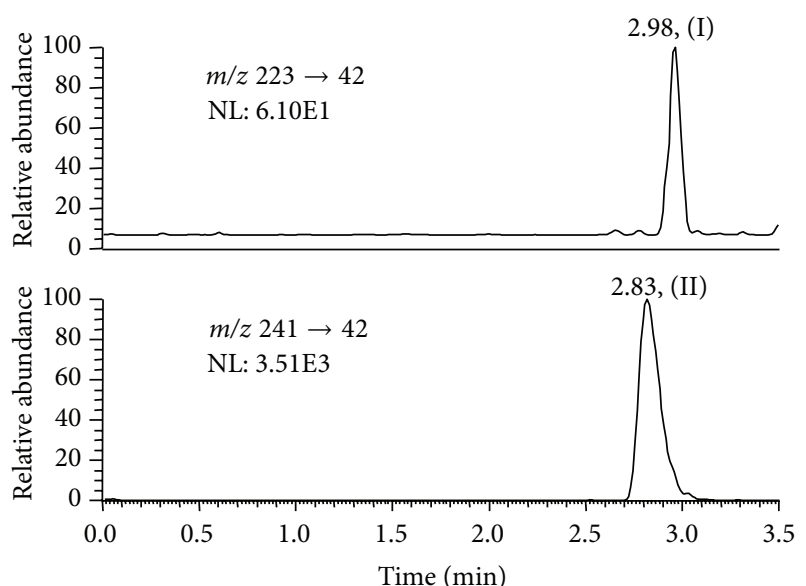

(b)

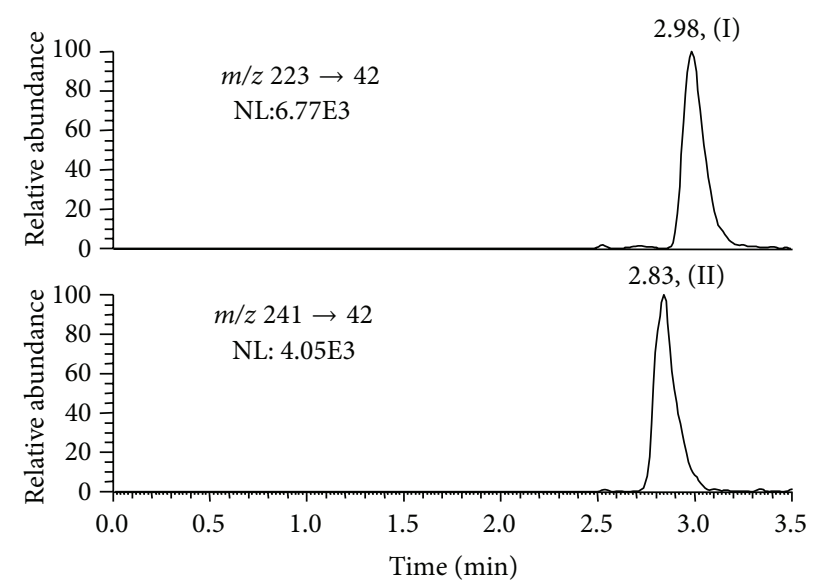

(c)

FIGURE 2: Representative selected reaction monitoring chromatograms of stavudine (I) and the internal standard (II). (a) Blank plasma sample; (b) plasma sample spiked with stavudine (LLOQ, $5.00 \mathrm{ng} / \mathrm{mL}$ ) and internal standard $(400 \mathrm{ng} / \mathrm{mL})$; (c) plasma sample from a volunteer $0.75 \mathrm{~h}$ after an oral dose of $20 \mathrm{mg}$ stavudine. 
TABLE 1: Precision and accuracy data for the analysis of stavudine.

\begin{tabular}{lcccr}
\hline Conc. added $(\mathrm{ng} / \mathrm{mL})$ & Conc. found $(\mathrm{ng} / \mathrm{mL})$ & Intraday RSD $(\%)$ & Interday RSD $(\%)$ & Accuracy $(\%)$ \\
\hline 5.00 & 5.12 & 6.1 & 0.9 & 102.5 \\
80.0 & 80.7 & 6.4 & 6.6 & 18 \\
900 & 902.2 & 2.9 & 9.7 & 180.8 \\
\hline
\end{tabular}

TABLE 2: Stability of stavudine.

\begin{tabular}{|c|c|c|c|c|c|}
\hline & Conc. added (ng/mL) & Conc. found (ng/mL) & RSD (\%) & Accuracy (\%) & $n$ \\
\hline \multirow{2}{*}{ Short-term stability } & 5.00 & 4.63 & 0.3 & 92.6 & 3 \\
\hline & 900 & 871 & 6.4 & 96.7 & 3 \\
\hline \multirow{2}{*}{ Postpreparative stability } & 5.00 & 4.61 & 0.3 & 92.2 & 3 \\
\hline & 900 & 831 & 6.2 & 92.3 & 3 \\
\hline
\end{tabular}

3.4. Linearity and LLOQ. According to stavudine plasma concentration levels of the preliminary experiment, the linear range was selected from $5.00 \mathrm{ng} / \mathrm{mL}$ to $1,000 \mathrm{ng} / \mathrm{mL}$. The LLOQ was $5.00 \mathrm{ng} / \mathrm{mL}$, which was more sensitive than the reported methods. The concentration of analyte was as the abscissa. The peak area ratio of analyte to the internal standard was as the ordinate. Using weighted least-squares method $\left(w=1 / x^{2}\right)[6]$, linear regression equation was calculated. A weighted least-squares $\left(w=1 / x^{2}\right)$ linear regression of response versus concentration was used for the calibration. A typical equation was as follows: $y=3.070 \times 10^{-3} x-1.129 \times$ $10^{-4}(r=0.9975)$. The RSD at the LLOQ was $6.1 \%$, and the accuracy was $102.5 \%$.

3.5. Precision and Accuracy. The accuracy and precision of the method were determined by assaying QC samples. The intra- and interbatch precisions and the accuracy were summarized in Table 1 . The results indicated that the values were within the acceptable range and the method was accurate and precise.

3.6. Extraction Recovery and Stability Study. The extraction recovery for stavudine was estimated at the three QC concentrations $(n=6)$. The extraction recoveries were $55.8 \%$, $54.3 \%$, and $54.4 \%$, respectively, and the recovery of the internal standard was $45.8 \%$. These data showed that the recovery values were similar and stable, and therefore no significant degradation occurred during the extraction and sample storage processes for stavudine in plasma samples.

The stability experiments were aimed at testing the effects of possible conditions that the analytes might experience during collection, storage, and analysis. It was reported that the stavudine plasma samples were stable at room temperature for $12 \mathrm{~h}$. Stavudine in plasma was stable at $-20^{\circ} \mathrm{C}$ for a period of 30 days and after three freeze $\left(-20^{\circ} \mathrm{C}\right.$ ) thaw (room temperature) cycles $[2,5]$. The short-term stability (at room temperature for $2 \mathrm{~h}$ ) and the postpreparative stability (at $20^{\circ} \mathrm{C}$ for $12 \mathrm{~h}$ ) were investigated. The data are demonstrated in Table 2 .

3.7. Bioequivalence and Pharmacokinetics Study. The mean plasma concentration-time curves were shown in Figure 3.



FIGURE 3: Mean plasma concentration versus time curve of stavudine after oral administration of $20 \mathrm{mg}$ stavudine capsule test and reference formulations to 18 healthy volunteers $(n=20, \bar{x} \pm s)$.

The main pharmacokinetic parameters are shown in Table 3. These data showed that $\mathrm{AUC}_{0-t}$ and $C_{\max }$ of stavudine reject not bioequivalent hypothesis. The test results of $T_{\max }$ nonparametric method showed that there was no significant difference between two formulations. Therefore, these two formulations were bioequivalent.

\section{Conclusions}

A LC-MS/MS method was established for determination of stavudine in human plasma. The linear range of stavudine plasma concentration was $5.00-1,000 \mathrm{ng} / \mathrm{mL}$. The LLOQ was $5.00 \mathrm{ng} / \mathrm{mL}$. Calculated by QC samples, the intra- and interassay precision (RSD) was less than $10 \%$. The accuracy was from $100.2 \%$ to $102.5 \%$. The results show that this method was rapid and sensitive, with good precision and accuracy. The separation time of every sample was $3.5 \mathrm{~min}$. Nearly 200 
TABLE 3: The main pharmacokinetic parameters.

\begin{tabular}{lccc}
\hline Parameter & Reference & Test & $90 \%$ confidence interval (\%) \\
\hline $\mathrm{AUC}_{0-t}(\mathrm{ng} \cdot \mathrm{h} / \mathrm{mL})$ & $872 \pm 228$ & $914 \pm 284$ & $98.6-109.8$ \\
$\mathrm{AUC}_{0-\infty}(\mathrm{ng} \cdot \mathrm{h} / \mathrm{mL})$ & $889 \pm 228$ & $931 \pm 287$ & \\
$C_{\max }(\mathrm{ng} / \mathrm{mL})$ & $480 \pm 151$ & $574 \pm 178$ & $97.8-125.1$ \\
$T_{\max }(\mathrm{h})$ & $0.55 \pm 0.19$ & $0.54 \pm 0.20$ & \\
$k_{e}\left(\mathrm{~h}^{-1}\right)$ & $0.42 \pm 0.09$ & $0.42 \pm 0.07$ & \\
$t_{1 / 2}(\mathrm{~h})$ & $1.72 \pm 0.43$ & $1.68 \pm 0.29$ & \\
$F(\%)$ & & $105.0 \pm 13.6$ & \\
\hline
\end{tabular}

plasma samples could be analyzed per day. This method was applied to human bioavailability and bioequivalence study of stavudine capsules.

\section{Conflict of Interests}

The author declares that there is no conflict of interests regarding the publication of this paper.

\section{Acknowledgments}

Financial support from the National Natural Science Foundation of China (Grant 51202213), Excellent Young Scientist Funding of Hebei Province (Grant Y2012005), and Qinhuangdao City Science \& Technology Research \& Development Program (no. 201001A120) is acknowledged.

\section{References}

[1] S. M. Cheer and K. L. Goa, "Stavudine once daily," Drugs, vol. 62, no. 18, pp. 2667-2674, 2002.

[2] M. Sarasa, N. Riba, L. Zamora, and X. Carne, "Determination of stavudine in human plasma and urine by high-performance liquid chromatography using a reduced sample volume," Journal of Chromatography B, vol. 746, no. 2, pp. 183-189, 2000.

[3] E. Kano, C.. dos Reis Serra, E. Koono, S. S. Andrade, and V. Porta, "Determination of lamivudine in human plasma by HPLC and its use in bioequivalence studies," International Journal of Pharmaceutics, vol. 297, no. 1-2, pp. 73-79, 2005.

[4] Z. Li, C. Ding, Q. Ge, Z. Zhou, X. Zhi, and X. Liu, "Simultaneous determination of lamivudine, stavudine and nevirapine in human plasma by LC-MS/MS and its application to pharmacokinetic study in clinic," Biomedical Chromatography, vol. 24, no. 9, pp. 926-934, 2010.

[5] J. L. Wiesner, F. C. W. Sutherland, M. J. Smit et al., "Sensitive and rapid liquid chromatography-tandem mass spectrometry method for the determination of stavudine in human plasma," Journal of Chromatography B, vol. 773, no. 2, pp. 129-134, 2002.

[6] D. Zhong, "Some aspects in establishing standard curves in bioanalyses with the weighted least squires method," Chinese Journal of Pharmaceutical Analysis, vol. 16, no. 5, pp. 343-346, 1996. 

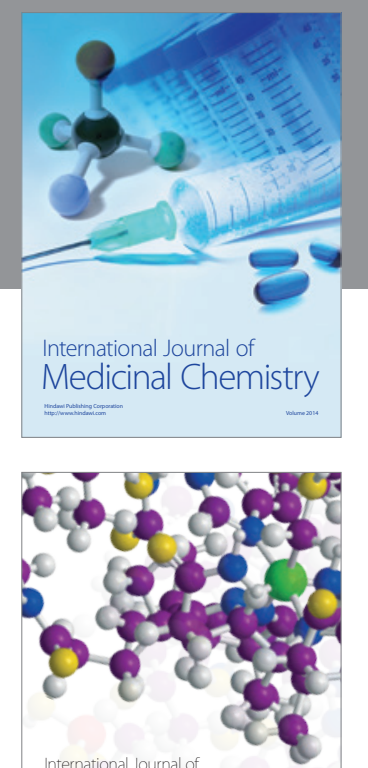

\section{Carbohydrate} Chemistry



The Scientific World Journal
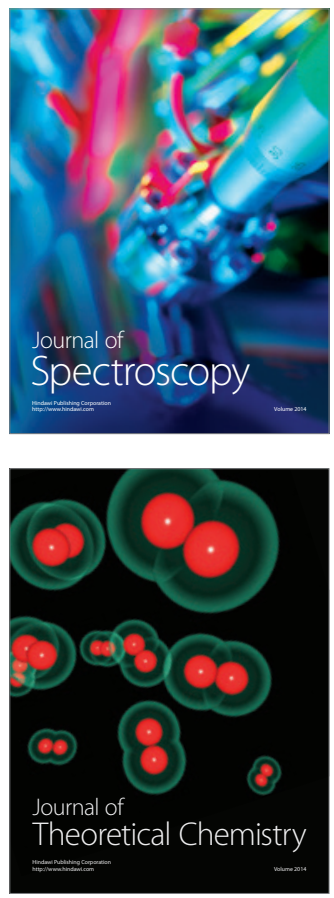
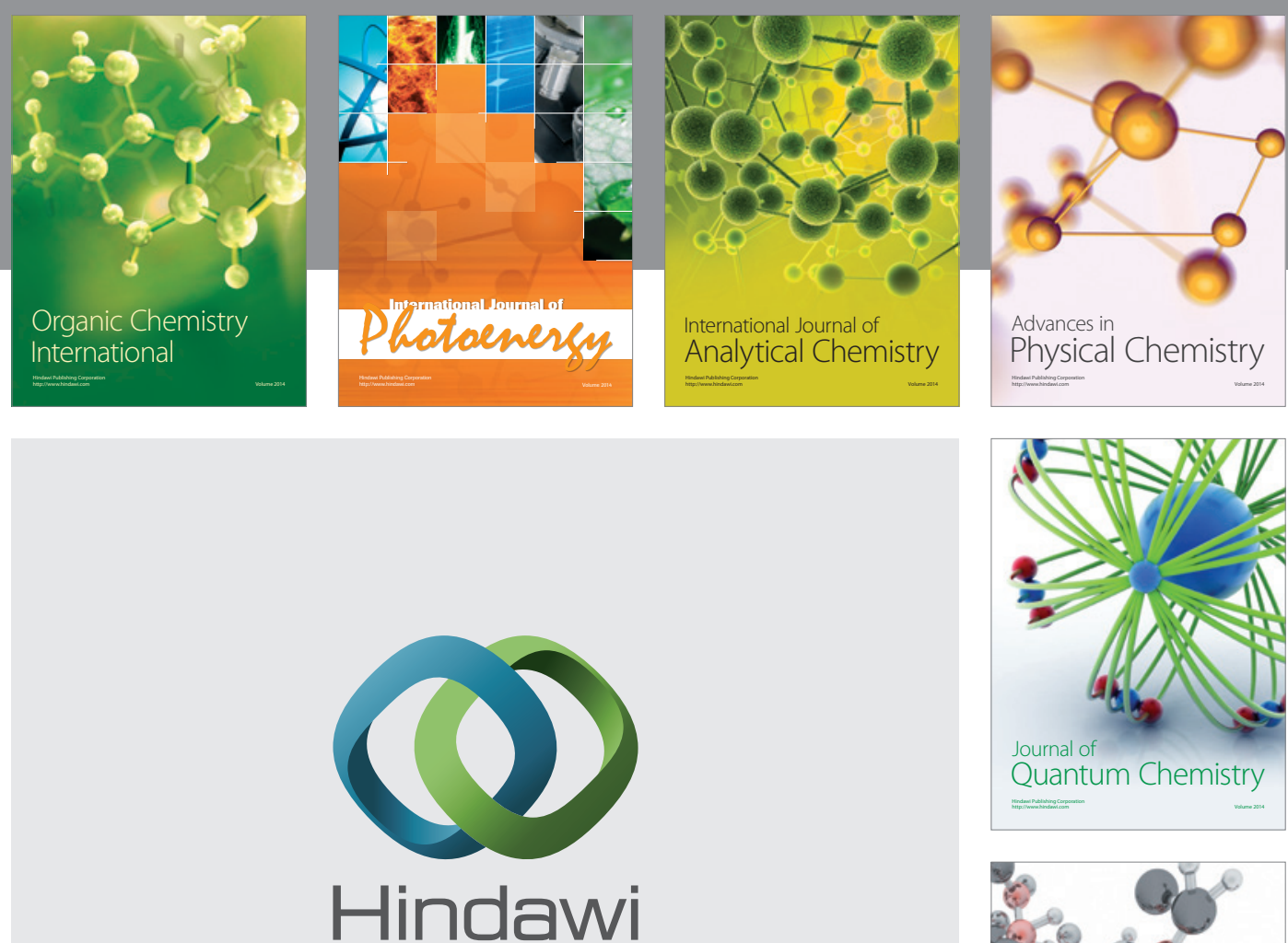

Submit your manuscripts at

http://www.hindawi.com

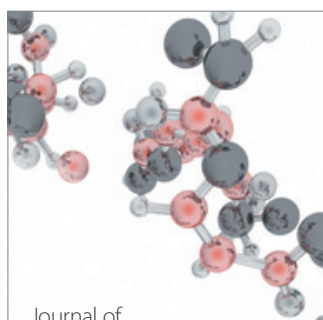

Analytical Methods

in Chemistry

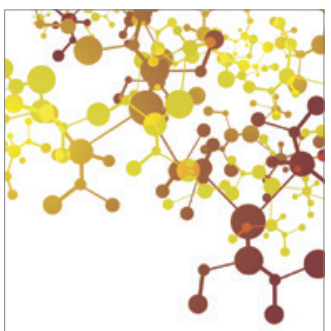

Journal of

Applied Chemistry



Inorganic Chemistry
